\title{
Relativistic descriptions of inclusive quasielastic electron scattering: Application to scaling and superscaling ideas
}

\author{
Andrea Meucci, ${ }^{1}$ J. A. Caballero, ${ }^{2}$ C. Giusti, ${ }^{1}$ F. D. Pacati, ${ }^{1}$ and J. M. Udías ${ }^{3}$ \\ ${ }^{1}$ Dipartimento di Fisica Nucleare e Teorica, Università degli Studi di Pavia and Istituto Nazionale di Fisica Nucleare, \\ Sezione di Pavia, I-27100 Pavia, Italy \\ ${ }^{2}$ Departamento de Física Atómica, Molecular y Nuclear, Universidad de Sevilla, E-41080 Sevilla, Spain \\ ${ }^{3}$ Grupo de Física Nuclear, Departamento de Física Atómica, Molecular y Nuclear, \\ Universidad Complutense de Madrid, E-28040 Madrid, Spain
}

(Received 15 June 2009; published 18 August 2009)

\begin{abstract}
An analysis of inclusive quasielastic electron scattering is presented using different descriptions of the final-state interactions within the framework of the relativistic impulse approximation. The relativistic Green's function approach is compared with calculations based on the use of relativistic purely real mean-field potentials in the final state. Both approaches lead to a redistribution of the strength but conserving the total flux. Results for the differential cross section at different energies are presented. Scaling properties are also analyzed and discussed.
\end{abstract}

DOI: 10.1103/PhysRevC.80.024605

PACS number(s): 24.10.Jv, 24.10.Cn, 25.30.Fj

\section{INTRODUCTION}

Electron-scattering reactions with nuclei have provided the most detailed and complete information on nuclear and nucleon structure. Analysis of data for light-to-heavy nuclei and for different kinematical situations have been presented in the literature [1-5]. Not only differential cross sections but also the contribution of the separate response functions have been considered. From the theoretical point of view, an important effort has been devoted for the past 20 years to the description of inclusive and exclusive processes. The high energies and momenta involved in recent experiments have led to the development of fully relativistic models describing the scattering process [6-17]. Moreover, ingredients beyond the quasielastic (QE) approach, namely meson exchange currents, correlations, etc., have been also considered following different approaches [18-32].

Within the QE domain, which is the region considered in this work, the treatment of final-state interactions (FSI) between the ejected nucleon and the residual nucleus has been proved to be essential to compare with data. This has been well established in the case of exclusive $\left(e, e^{\prime} N\right)$ reactions where an ejected nucleon is detected in coincidence with the scattered electron. Analyses based on the use of phenomenological complex potentials in the final channel have provided results in accordance with data $[1,4]$. In particular, the use of relativistic complex optical potentials within a fully relativistic description of the reaction mechanism has led to theoretical cross sections in excellent accordance with data $[7,8,10,11,13,15,16]$. Moreover, comparison with separate responses and asymmetries has also proved the capability of the relativistic approach to successfully describe fine details of data behavior $[9,10,13,33,34]$.

In the analysis of inclusive reactions, contrary to exclusive ones, all inelastic channels in the final state should be retained. This means that the flux is conserved, and, consequently, the distorted-wave impulse approximation (DWIA) based on the use of a complex potential should be dismissed. However, final-state interaction continues to be a main ingredient in the inclusive process, and its appropriate description is required to describe data. Within the framework of fully relativistic models different approaches have been presented in the literature. First, a description based on the relativistic distorted-wave impulse approximation (RDWIA) has been pursued but using purely real potentials in the final channel. This is consistent with flux conservation. Concerning the specific potentials, the final nucleon state has been evaluated with the real part of the relativistic energy-dependent optical potential, denoted as rROP, or with the same relativistic mean-field potential considered in describing the initial nucleon state, denoted as RMF (see Refs. [15-17,35]). A second approach of FSI makes use of the relativistic Green's function technique, where the components of the nuclear response are written in terms of the single-particle optical model Green's function. This method, denoted as GF, allows one to perform calculations treating FSI consistently in the inclusive and exclusive channels. The same relativistic (complex) optical potential is considered in both cases, but flux is conserved in the inclusive process. Moreover, redistribution of strength among different channels is due to the real and also significantly to the imaginary part of the potential (see Refs. [14,36-39] for details).

The exhaustive analysis of the $\left(e, e^{\prime}\right)$ world data has demonstrated the quality of scaling arguments at high-momentum transfer for excitation energies below the QE peak [40-43]. These data, and particularly those coming from the separate longitudinal contribution, have been shown to respect scaling of first kind (independence of the transferred momentum $q$ ) and scaling of second kind (no dependence on the nuclear system). The analysis of the longitudinal response, very mildly affected by meson exchange currents and nuclear correlations, has permitted the extraction of a phenomenological QE superscaling function given for transferred energies below and above the QE peak. These regions correspond to negative and positive values of the so-called superscaling variable, respectively [35,41-44]. Scaling analysis for electron scattering, which was extended into the $\Delta$ region $[20,45,46]$, has been also exploited to predict inclusive charged-current 
(CC) neutrino-nucleus cross sections [35,44,45,47-50] and neutral-current (NC) processes [51-53].

The QE phenomenological scaling function presents a significant asymmetry with a tail extended to high values of the transferred energy $\omega$ [positive values of the scaling variable $\psi^{\prime}(q, \omega)$ ]. This imposes strong restrictions to all theoretical models describing $\mathrm{QE}\left(e, e^{\prime}\right)$ processes, namely they should be able to fulfill scaling properties and in addition reproduce the specific shape of the scaling function. Asymmetry in the scaling function is largely absent in nonrelativistic models based on a mean-field approach [48]. The same comment applies to the relativistic plane wave limit and to results based on a DWIA using real relativistic energy-dependent optical potentials $[35,44]$. In contrast, asymmetry comes out within the relativistic impulse approximation but with FSI described using strong relativistic mean-field potentials. An asymmetrical scaling function has been also shown to occur within the framework of a semirelativistic model, based on FSI given through the Dirac equation-based (DEB) potential derived from the RMF (see Ref. [54] for details).

Previous studies clearly illustrate the central role played by FSI in providing theoretical results in accordance with data. Hence, in this work we present a careful analysis of $\left(e, e^{\prime}\right)$ processes comparing two basic descriptions of FSI: the RMF approach and the relativistic GF technique. Both models have their own merits. In the RMF the flux lost into inelasticities is recovered by using the same, purely real, energy-independent potential seen by the bound nucleons and thus no information from scattering reactions is explicitly incorporated in the model. In this way it is consistent with dispersion relations [55]. The RMF leads to an asymmetrical scaling function that is supported on overall by data behavior. However, it breaks down scaling significantly as the transferred momentum $q$ increases, particularly in the region above the $\mathrm{QE}$ peak. The analysis of experimental $\left(e, e^{\prime}\right)$ data indeed leaves room for first-kind scaling breaking in this region, due partly to $\Delta$ production and other contributions beyond the impulse approximation, namely meson exchange currents and their impact in the $2 p$ - $2 h$ sector [24]. The GF approach provides a consistent and unified treatment of inclusive and exclusive electron-nucleus scattering processes. It also fulfills dispersion relations and recovers the flux lost into inelasticities by means of a formal summation of unobserved channels performed via the optical potential. Most often, the optical potential is taken from phenomenology, and thus, in this approach, information on the observed nucleon-nucleus scattering is incorporated into the model.

The differences observed between the predictions of the two approaches and their behavior with regard to scaling arguments will be helpful in establishing an appropriate description of FSI for inclusive $\left(e, e^{\prime}\right)$ processes at different kinematics. Further, this would be useful for understanding nuclear effects, especially FSI, in neutrino-nucleus cross sections [35,37,44,45,56-65], which are of paramount interest for the ongoing and future neutrino oscillation experiments [66-74].

Therefore, in this work we present a systematic study of both models, comparing their predictions and analyzing their scaling properties, with special emphasis on the specific shape of the scaling function.

The article is organized as follows. In Sec. II, we present the basic formalism involved in inclusive quasielastic electron scattering with a discussion of the different models considered in the description of FSI, namely the relativistic mean field and the relativistic Green's function approach. We also provide a subsection that contains the basic facts related to scaling arguments and introduce superscaling functions. In Sec. III, we show and discuss our results. First, we focus on the analysis of differential cross sections and later we analyze in detail results for the scaling function. Finally, in Sec. IV we summarize the main results and present our conclusions.

\section{INCLUSIVE QUASIELASTIC ELECTRON SCATTERING}

An electron with four-momentum $K^{\mu}=(\varepsilon, \boldsymbol{k})$ is scattered through an angle $\vartheta_{e}$ to four-momentum $K^{\prime \mu}=\left(\varepsilon^{\prime}, \boldsymbol{k}^{\prime}\right)$. The four-momentum transfer is $Q^{\mu}=K^{\mu}-K^{\prime \mu}=(\omega, \boldsymbol{q})$. In the one-photon exchange approximation the inclusive cross section for the quasielastic $\left(e, e^{\prime}\right)$ scattering on a nucleus is given by [1]

$$
\left(\frac{d \sigma}{d \varepsilon^{\prime} d \Omega^{\prime}}\right)=\sigma_{M}\left[V_{L} R_{L}+V_{T} R_{T}\right],
$$

where $\Omega^{\prime}$ is the scattered electron solid angle and $\sigma_{M}$ is the Mott cross section [1]. The coefficients $V$ come from the lepton tensor components and are defined as

$$
V_{L}=\left(\frac{\left|Q^{2}\right|}{|\boldsymbol{q}|^{2}}\right)^{2} V_{T}=\tan ^{2} \frac{\vartheta_{e}}{2}-\frac{\left|Q^{2}\right|}{2|\boldsymbol{q}|^{2}},
$$

where $\left|Q^{2}\right|=|\boldsymbol{q}|^{2}-\omega^{2}$.

All nuclear structure information is contained in the longitudinal and transverse response functions, $R_{L}$ and $R_{T}$, expressed by

$$
\begin{aligned}
& R_{L}(q, \omega)=W^{00}(q, \omega) \\
& R_{T}(q, \omega)=W^{11}(q, \omega)+W^{22}(q, \omega)
\end{aligned}
$$

in terms of the diagonal components of the hadron tensor that is given by bilinear products of the transition matrix elements of the nuclear electromagnetic many-body current operator $\hat{J}^{\mu}$ between the initial state $\left|\Psi_{0}\right\rangle$ of the nucleus, of energy $E_{0}$, and the final states $\left|\Psi_{f}\right\rangle$, of energy $E_{f}$, both eigenstates of the $(A+1)$-body Hamiltonian $H$, as

$$
\begin{aligned}
W^{\mu \nu}(q, \omega)= & \sum_{i} \oint_{f}\left\langle\Psi_{f}\left|\hat{J}^{\mu}(\boldsymbol{q})\right| \Psi_{0}\right\rangle \\
& \times\left\langle\Psi_{0}\left|\hat{J}^{\nu \dagger}(\boldsymbol{q})\right| \Psi_{f}\right\rangle \delta\left(E_{0}+\omega-E_{f}\right),
\end{aligned}
$$

involving an average over the initial states and a sum over the undetected final states. The sum runs over the scattering states corresponding to all of the allowed asymptotic configurations and includes possible discrete states.

In the $\mathrm{QE}$ region the nuclear response is dominated by one-nucleon knockout processes, where the scattering occurs with only one nucleon that is subsequently emitted. The remaining nucleons of the target behave as simple spectators. 
Therefore, QE electron scattering is adequately described in the relativistic impulse approximation (RIA) by the sum of incoherent processes involving only one nucleon scattering.

In the RIA the nuclear current operator is assumed to be the sum of single-nucleon currents $\hat{j}^{\mu}$, for which different relativistic free nucleon expressions [75] can be used. In the present calculations we use the option denoted as $\mathrm{CC} 2$, i.e.,

$$
\hat{j}^{\mu}=F_{1}\left(Q^{2}\right) \gamma^{\mu}+i \frac{\kappa}{2 M} F_{2}\left(Q^{2}\right) \sigma^{\mu \nu} Q_{\nu},
$$

where $\kappa$ is the anomalous part of the magnetic moment and $\sigma^{\mu \nu}=(i / 2)\left[\gamma^{\mu}, \gamma^{\nu}\right] . F_{1}$ and $F_{2}$ are the Dirac and Pauli nucleon form factors [76].

Within the RIA framework and under the assumption of a shell-model description for nuclear structure, the components of the hadron tensor are obtained from the sum, over all the single-particle (s.p.) shell-model states, of the squared absolute value of the transition matrix elements of the single-nucleon current

$$
\left\langle\chi_{E}^{(-)}(E)\left|\hat{j}^{\mu}(\boldsymbol{q})\right| \varphi_{n}\right\rangle,
$$

where $\chi_{E}^{(-)}(E)$ is the scattering state of the emitted nucleon and the overlap $\varphi_{n}$ between the ground state of the target $\left|\Psi_{0}\right\rangle$ and the final state $|n\rangle$ of the residual nucleus is a single-particle shell-model state.

In the calculations presented in this work the bound nucleon states $\varphi_{n}$ are taken as self-consistent Dirac-Hartree solutions derived within a RMF approach using a Lagrangian containing $\sigma, \omega$, and $\rho$ mesons [77-81].

Different prescriptions are used to calculate the relativistic s.p. scattering wave functions. In the simplest approach the plane-wave limit is considered, i.e., the relativistic planewave impulse approximation (RPWIA), where FSI between the outgoing nucleon and the residual nucleus are neglected. In the approaches based on the relativistic distorted-wave impulse approximation FSI effects are accounted for by solving the Dirac equation with relativistic optical potentials. The use of relativistic energy-dependent complex optical potentials fitted to elastic proton-nucleus scattering data has been very successful in describing exclusive $\left(e, e^{\prime} p\right)$ data $[1,4,7-13]$. In the exclusive scattering the imaginary part of the optical potential produces an absorption that reduces the calculated cross section and accounts for the flux lost in the considered elastic channel toward other channels. This approach is appropriate for the exclusive process where only one channel is considered, but it would be inconsistent for the inclusive scattering, where all the inelastic channels should be retained and the total flux, although redistributed among all possible channels due to FSI, must be conserved. As a result, in the RDWIA (with complex potentials) the flux is not conserved and the inclusive $\left(e, e^{\prime}\right)$ cross section is underestimated [15-17,36]. A simple way of estimating the inclusive response within the RIA, avoiding spurious flux absorption, is to use purely real potentials. In a first approach, the imaginary part of the phenomenological relativistic energy-dependent optical potentials [82] is set to zero, thus retaining in the calculations only the real part. In a second approach, the scattering states are described by using the same RMF theory potentials considered in the description of the initial bound state $\varphi_{n}$. We refer to these two different
FSI descriptions as real relativistic optical potential (rROP) and relativistic mean field (RMF), respectively.

We note that rROP conserves the flux and thus it is inconsistent with the exclusive process, where a complex optical potential must be used. Moreover, the use of a real optical potential is unsatisfactory from a theoretical point of view, because it is an energy-dependent potential, reflecting the different contribution of open inelastic channels for each energy. Dispersion relation then dictates that the potential should have a nonzero imaginary term [55]. However, the RMF model is based on the use of the same strong energyindependent real potential for both bound and scattering states, so it fulfills the dispersion relation [55] and, further, it maintains the continuity equation.

Green's function techniques are exploited to derive the inclusive response in a different model where the flux is conserved and the use of a complex optical potential allows one to treat FSI consistently in the inclusive and in the exclusive reactions. Detailed discussions of this Green's function (GF) approach can be found in Refs. [14,36-39]. Here we recall only the essential features of the model.

In the GF approach the components of the nuclear response in Eq. (4) are written in terms of the s.p. optical model Green's function. This is the result of suitable approximations, such as the one-body current assumption and subtler approximations related to the IA. The explicit calculation of the s.p. Green's function can be avoided by its spectral representation, which is based on a biorthogonal expansion in terms of a non-Hermitian optical potential and of its Hermitian conjugate. The nuclear response of Eq. (4) is then obtained in the form [36]

$$
\begin{aligned}
W^{\mu \mu}(q, \omega)= & \sum_{n}\left[\operatorname{Re} T_{n}^{\mu \mu}\left(E_{f}-\varepsilon_{n}, E_{f}-\varepsilon_{n}\right)\right. \\
& -\frac{1}{\pi} \mathcal{P} \int_{M}^{\infty} d \mathcal{E} \frac{1}{E_{f}-\varepsilon_{n}-\mathcal{E}} \\
& \left.\times \operatorname{Im} T_{n}^{\mu \mu}\left(\mathcal{E}, E_{f}-\varepsilon_{n}\right)\right]
\end{aligned}
$$

where $n$ denotes the eigenstate of the residual nucleus related to the discrete eigenvalue $\varepsilon_{n}$ and

$$
\begin{aligned}
T_{n}^{\mu \mu}(\mathcal{E}, E)= & \left\langle\varphi_{n}\left|\hat{j}^{\mu \dagger}(\boldsymbol{q}) \sqrt{1-\mathcal{V}^{\prime}(E)}\right| \tilde{\chi}_{\mathcal{E}}^{(-)}(E)\right\rangle \\
& \times\left\langle\chi_{\mathcal{E}}^{(-)}(E)\left|\sqrt{1-\mathcal{V}^{\prime}(E)} \hat{j}^{\mu}(\boldsymbol{q})\right| \varphi_{n}\right\rangle .
\end{aligned}
$$

The factor $\sqrt{1-\mathcal{V}^{\prime}(E)}$, where $\mathcal{V}^{\prime}(E)$ is the energy derivative of the optical potential, accounts for interference effects between different channels and justifies the replacement in the calculations of the Feshbach optical potential $\mathcal{V}$ of the GF model, for which neither microscopic nor empirical calculations are available, by the local phenomenological optical potential $[36,38]$. Disregarding the square-root correction, the second matrix element in Eq. (8) is the transition amplitude of single-nucleon knockout of Eq. (6), where the imaginary part of the optical potential accounts for the flux lost in the channel $n$ toward the channels different from $n$. In the inclusive response this loss is compensated by a corresponding gain of flux due to the flux lost, toward the channel $n$, by the other final states asymptotically originated by the channels different from $n$. This compensation is performed by the first matrix element 
in the right-hand side of Eq. (8) that is similar to the matrix element of Eq. (6) but involves the eigenfunction $\tilde{\chi}_{\mathcal{E}}^{(-)}(E)$ of the Hermitian conjugate optical potential, where the imaginary part has an opposite sign and has the effect of increasing the strength. In the GF approach the imaginary part of the optical potential redistributes the flux lost in a channel in the other channels, and in the sum over $n$ the total flux is conserved.

The hadron tensor in Eq. (7) is the sum of two terms. The calculation of the second term requires integration over all the eigenfunctions of the continuum spectrum of the optical potential. If the imaginary part of the optical potential is neglected, the second term in Eq. (7) vanishes and, but for the square root factor (whose contribution is however generally small in the calculations), the first term gives the rROP approach.

\section{A. Scaling at the quasielastic peak}

Scaling ideas applied to inclusive QE electron-nucleusscattering reactions have been shown to work properly to high accuracy [41-43]. In fact, an exhaustive analysis of QE $\left(e, e^{\prime}\right)$ world data has demonstrated that scaling property is well respected at high-momentum transfer for excitation energies falling below the QE peak. These data, when plotted against a properly chosen variable (the scaling variable), show a mild dependence on the momentum transfer (reasonable scaling of first kind) and almost no dependence on the nuclear target (excellent scaling of second kind). Simultaneous fulfillment of both conditions leads to superscaling. The analysis of the separated longitudinal contribution to $\left(e, e^{\prime}\right)$ data has led to introduce a phenomenological scaling function that contains the relevant information about the nuclear dynamics explored by the probe. Scaling and superscaling are general phenomena exhibited by nature which any "reliable" model providing a description of the scattering process should be able to reproduce. Not only superscaling behavior should be described but also the specific magnitude and shape of the "universal" phenomenological superscaling function needs to be reproduced.

The usual procedure to get the scaling function consists of dividing the inclusive differential cross section (1) by the appropriate single-nucleon $e N$ elastic cross section weighted by the corresponding proton and neutron numbers $[41-43,83]$ involved in the process,

$$
f\left(\psi^{\prime}, q\right) \equiv k_{F} \frac{\left[\frac{d \sigma}{d \varepsilon^{\prime} d \Omega^{\prime}}\right]}{\sigma_{M}\left[V_{L} G_{L}(q, \omega)+V_{T} G_{T}(q, \omega)\right]} .
$$

Analogously, the analysis of the separated longitudinal $(L)$ and transverse $(T)$ contributions leads to scaling functions,

$$
f_{L(T)}\left(\psi^{\prime}, q\right) \equiv k_{F} \frac{R_{L(T)}(q, \omega)}{G_{L(T)}(q, \omega)} .
$$

The term $\psi^{\prime}(q, \omega)$ is the dimensionless scaling variable extracted from the relativistic Fermi gas (RFG) analysis that incorporates the typical momentum scale for the selected nucleus [35,41]. The fully relativistic expressions for $G_{L(T)}$ involve the proton and neutron form factors $G_{E(M)}^{p n}$, weighted by the proton and neutron numbers, and an additional dependence on the nuclear scale given through the Fermi momentum $k_{F}$ (explicit expressions are given by Eqs. (16)-(19) in Ref. [41]).

Whereas $L$ data are compatible with superscaling behavior, permitting the extraction of the phenomenological function $f_{L}^{\exp }\left(\psi^{\prime}\right)$, scaling is known to be violated in the $T$ channel at energies above the QE peak by effects beyond the impulse approximation [20-24,84]. It is important to point out that, although many models based on the impulse approximation exhibit superscaling, even perfectly as the RFG, only a few of them are capable to accurately reproduce the asymmetric shape of $f_{L}^{\text {exp }}$ with a significant tail extended to high transferred energies (large positive values of the scaling variable $\psi^{\prime}$ ). One of these is based on the RIA with final-state interactions described by means of strong relativistic mean-field potentials. On the contrary, calculations based on the plane wave limit and/or the use of real relativistic optical potentials in the final state, although satisfying superscaling properties, lead to "symmetrical-shape" scaling functions that are not in accordance with data analysis $[35,44,47]$.

In this work we extend the analysis of scaling to the relativistic GF approach, whose predictions will be compared with previous results obtained within the RMF, RPWIA, and rROP frameworks. This study will show to which degree superscaling is fulfilled by the GF calculations, and, moreover, how the GF scaling function compares with the experiment. As already mentioned, the GF treats FSI consistently in the inclusive and exclusive reactions, hence its application to scaling and superscaling ideas, which emerge as an essential outcome of nature, results mandatory. This allows us to get a clear insight concerning the capability of the relativistic GF approach applied to $\mathrm{QE}$ inclusive $\left(e, e^{\prime}\right)$ reactions, as well as the uncertainties ascribed to the various ingredients of the model, particularly the specific energy-dependent terms in the complex optical potentials involved.

\section{RESULTS AND DISCUSSION}

In this section the numerical results of the different relativistic models developed by the Pavia and the MadridSevilla groups to describe FSI in the inclusive quasielastic electron-nucleus scattering are considered. As a first step results obtained by the two groups in the RPWIA and rROP approaches are compared to check the consistency of the numerical programs when calculations are carried out under the same conditions. Then the results corresponding to the RMF model developed by the Madrid-Sevilla group and the relativistic GF model developed by the Pavia group are compared. This comparison is performed for the ${ }^{12} \mathrm{C}\left(e, e^{\prime}\right)$ cross section and scaling function calculated with various models under different kinematics.

\section{A. Differential cross section}

The ${ }^{12} \mathrm{C}\left(e, e^{\prime}\right)$ cross section has been calculated for a fixed value of the incident electron energy $\varepsilon=1 \mathrm{GeV}$ and three values of the momentum transfer, i.e., $q=500,800$, and $1000 \mathrm{MeV} / c$. The relativistic initial states are taken as 

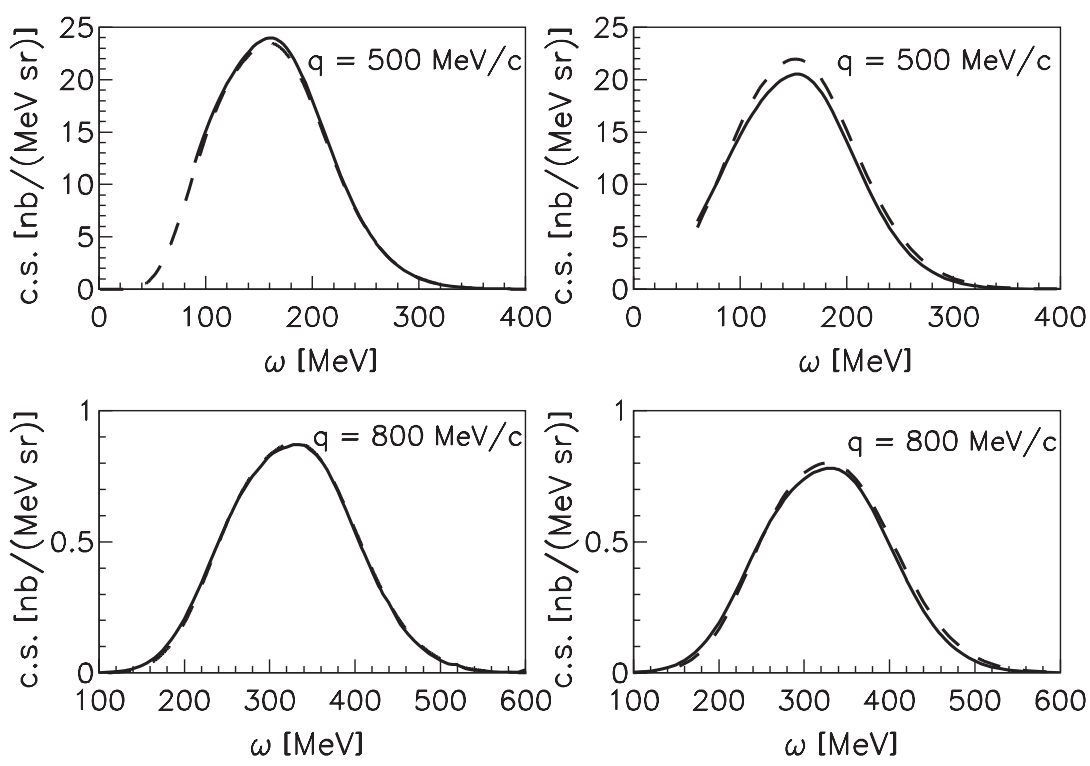

FIG. 1. Differential cross section of the ${ }^{12} \mathrm{C}\left(e, e^{\prime}\right)$ reaction for an incident electron energy $\varepsilon=1 \mathrm{GeV}$ and three values of the momentum transfer, i.e., $q=500,800$, and $1000 \mathrm{MeV} / c$, calculated by the Pavia (solid lines) and the Madrid-Sevilla (dashed lines) groups with RPWIA (left column) and rROP (right column).

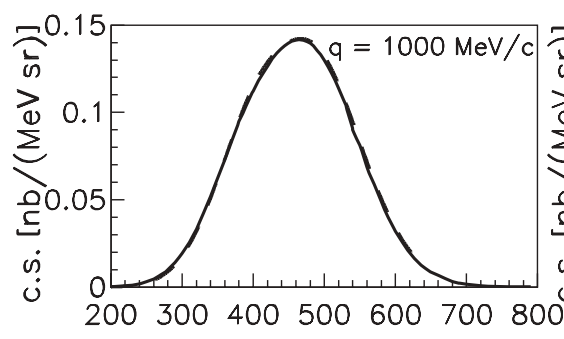

$\omega[\mathrm{MeV}]$

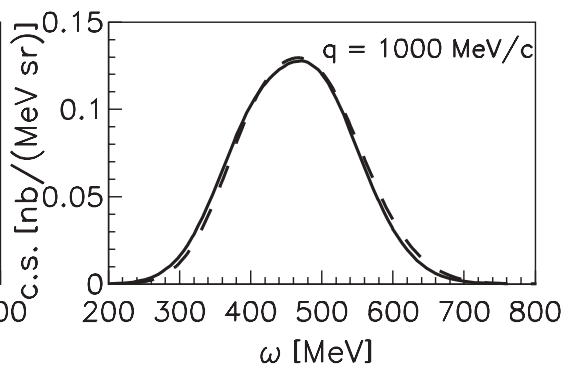

Dirac-Hartree solutions of a relativistic Lagrangian written in the context of a relativistic mean-field theory with the NLSH parametrization [79-81].

The cross sections calculated in RPWIA and in rROP by the Pavia and the Madrid-Sevilla groups are compared in Fig. 1. The calculations are performed using the same ingredients for the relativistic wave functions and the current operator. Almost identical results are obtained in RPWIA. In rROP the two results are very similar, up to a few percentages.

The comparison in Fig. 1 is a first important and necessary benchmark of the two independent computer programs, which allows us to estimate the numerical uncertainties and gives enough confidence on the reliability of both calculations. Further discrepancies between the results of the two groups can now be ascribed to differences of the models but not to inconsistencies in the calculations.

The cross sections evaluated with the RMF and GF models for the same kinematics as in Fig. 1 are presented in Fig. 2. In the case of the GF approach two different parametrizations for the relativistic optical potential have been used: the energy-dependent and $A$-dependent EDAD1 and EDAD2 complex phenomenological potentials of Ref. [82], which are fitted to proton elastic-scattering data on several nuclei in an energy range up to $1040 \mathrm{MeV}$. In the figures the results obtained with EDAD1 and EDAD2 are denoted as GF1 and GF2, respectively. The differences between the results of the RMF and GF models increase with the momentum transfer. Moreover, discrepancies between the GF1 and GF2 cross sections depend on the momentum transfer. At

$q=500 \mathrm{MeV} / c$ the three results in Fig. 2 are similar, both in magnitude and shape. At larger $q, q=800 \mathrm{MeV} / c$, moderate differences are found, whereas the discrepancy between the three approaches gets larger at $q=1000 \mathrm{MeV} / c$. The shape of the RMF cross section shows an asymmetry, with a long tail extending toward higher values of $\omega$ that is essentially due to the strong energy-independent scalar and vector potentials present in the RMF approach. On the contrary, in the case of GF1 and GF2, the asymmetry toward higher $\omega$ is less significant but still visible. The GF1 and GF2 cross sections show a similar shape but with a significant difference in the magnitude. At $q=1000 \mathrm{MeV} / c$ both of them are higher than the RMF cross section in the region where the maximum occurs. However, note that a stronger enhancement is obtained with GF1, which at the peak overshoots the RMF cross section up to $40 \%$. Overall, taking into account the different ingredients that these estimates of the inclusive response contain, the similarity of the predictions for the inclusive cross section is remarkable, particularly at the two lower values of $q$. The larger differences seen for the largest $q$ value, not only between the RMF and GF models but also between the two GF results, is simply an indication of the difference in the ingredients of these calculations.

Indeed, the different behavior presented by the RMF and GF results as a function of $q$ and $\omega$ is directly linked to the specific structure of the relativistic potentials involved in the RMF and GF models. Whereas the RMF is based on the use of a strong energy-independent real potential, the GF approach makes use of a complex energy-dependent 

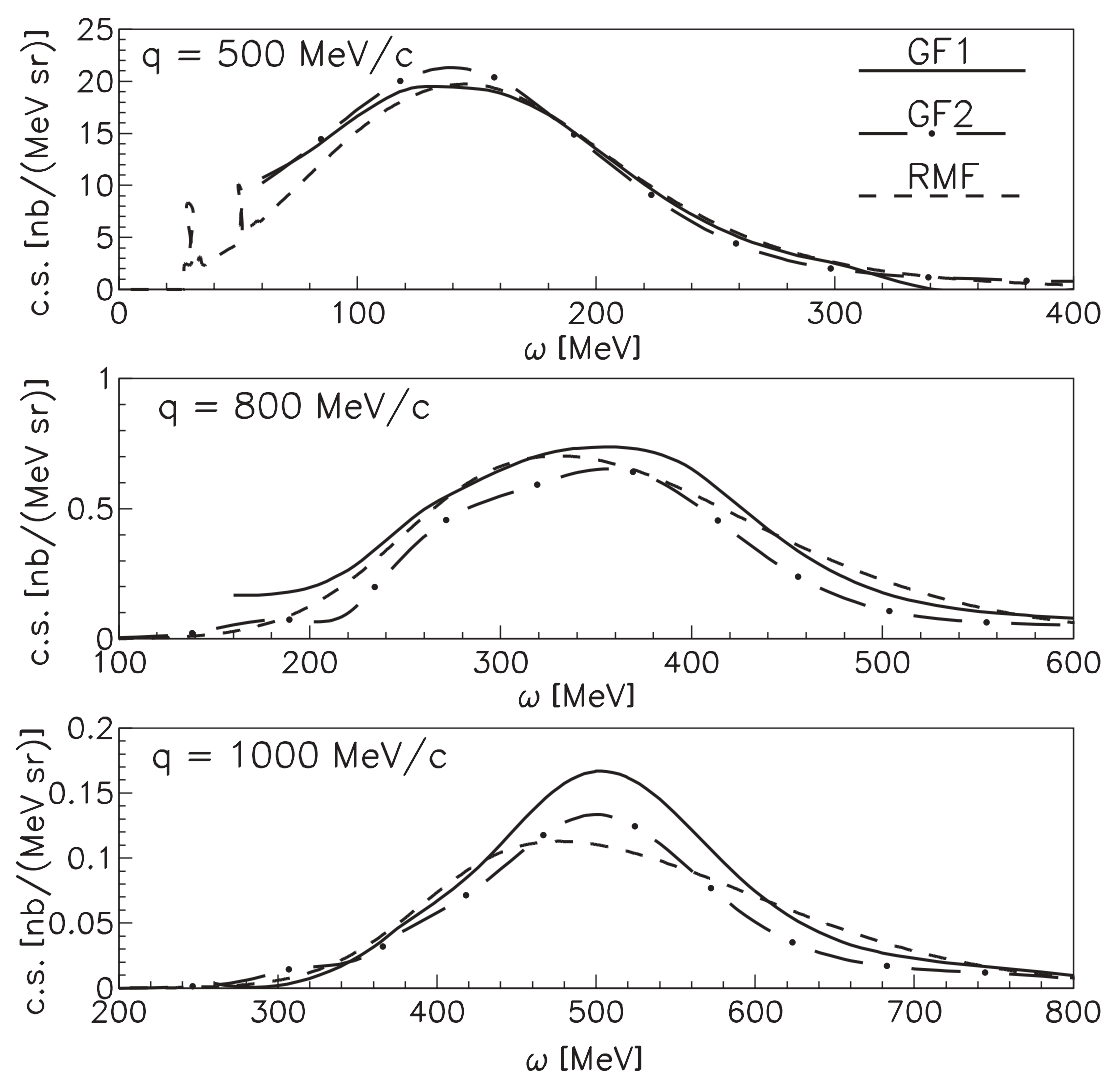

FIG. 2. Differential cross section of the ${ }^{12} \mathrm{C}\left(e, e^{\prime}\right)$ reaction in the same kinematics as in Fig. 1. The solid and long dot-dashed lines are the GF results calculated with the two different optical potentials EDAD1 and EDAD2. The dashed lines are the results of the RMF model. optical potential. In GF calculations the behavior of the optical potential changes with the momentum and energy transferred in the process, and higher values of $q$ and $\omega$ correspond to higher energies for the optical potential. The results obtained with GF1 and GF2 are consistent with the general behavior of the phenomenological relativistic optical potentials and are basically due to their imaginary part. To make this clear, we present again in Fig. 3 the GF and RMF cross sections for $q=500$ and $1000 \mathrm{MeV} / c$ compared directly with the corresponding results obtained within the RPWIA and rROP models. It is known that the real terms of the relativistic optical potentials are very similar for the different parametrizations. This explains why the cross section evaluated within the rROP approach does not show sensitivity to the particular parametrization considered for the ROP. However, the energydependent scalar and vector components of the real part of the ROP get smaller with increasing energies. Thus the rROP result approaches the RPWIA one for large values of $\omega$. In contrast, the imaginary (scalar and vector) part presents its maximum strength around $500 \mathrm{MeV}$ being also sensitive to the particular ROP parametrizations. This explains the differences observed between the rROP and the two GF results as a function of $\omega$ and $q$. This significant discrepancy between GF and rROP cross sections in Fig. 3 seems to contradict previous results shown in Ref. [37]. However, kinematical conditions reported in Ref. [37] corresponded to lower values of the momentum transfer, where there is no reason a priori to expect the rROP and GF predictions to be closer or further apart.

Of particular relevance is the difference between the GF1 and GF2 results. These are obtained with optical potentials that reproduce the elastic proton-nucleus phenomenology to a similar degree [82]. However, one must keep in mind that elastic observables do not completely constrain optical potentials, and indeed it has been often seen how the predictions of the EDAD1 and EDAD2 potentials for nonelastic observables, such as for instance $\left(e, e^{\prime} p\right)$ or electron trasparencies [56,57], differ significantly. The differences between GF1 and GF2 are mostly due to their different imaginary part, that includes the overall effect of the inelastic channels and is not univocally determined by the elastic phenomenology. The most convenient choice of the phenomelogical optical potential to be employed within the GF approach should thus be made after a comparison to inclusive data.

In Fig. 4 the GF1, GF2, and RMF results are compared with the experimental cross sections for three different kinematics [85-87]. A recent review of the experimental situation as well as different theoretical approaches can be found in Ref. [2]. Results in Fig. 4 show that the three models lead to similar cross sections. The main differences are presented for higher values of the momentum transfer, about $800 \mathrm{MeV} / c$ (bottom panel), where the GF1 cross section (solid line) is larger than the GF2 (dot-dashed) and RMF (dashed) ones. The experimental cross section is slightly underpredicted in the top panel and well described in the middle panel by all calculations. Finally, results in the bottom panel show a fair agreement with data in the case of GF1, whereas GF2 and RMF underpredict the experiment. Summarizing, the comparison with data, although satisfactory on general grounds, gives only an indication and cannot be conclusive until contributions beyond the QE peak, like meson exchange currents and Delta effects, which may play a significant role in the analysis of data even at the maximum of the QE peak, are carefully 

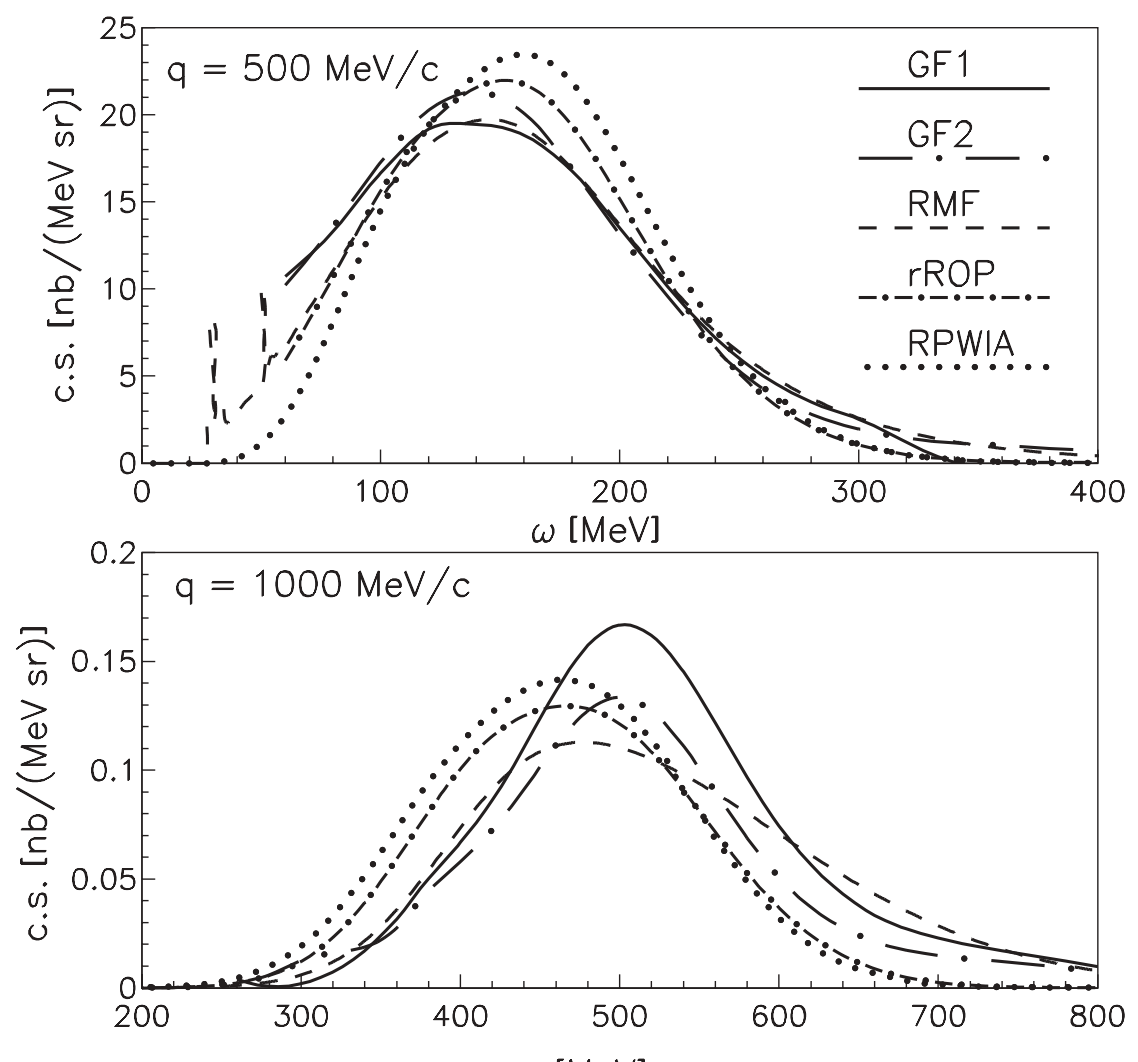

FIG. 3. Differential cross section of the ${ }^{12} \mathrm{C}\left(e, e^{\prime}\right)$ reaction for $\varepsilon=1 \mathrm{GeV}$ and $q=500$ and $1000 \mathrm{MeV} / c$. The solid, long dot-dashed, and dashed lines are the same as in Fig. 2. The dot-dashed and dotted lines are the rROP and RPWIA results, respectively. evaluated $[20,45,46]$. These processes contribute to the cross section and the comparison of the pure nucleonic predictions of the GF1, GF2, and RMF models to data will only indicate what is the contribution of the non-nucleonic degrees of freedom to the cross sections.

\section{B. Scaling functions}

The effects already discussed for the differential cross sections are also present in the scaling functions. Here we compare results for the longitudinal component of the scaling function $f_{L}\left(\psi^{\prime}\right)$ using the same descriptions for the finalstate interactions already considered for the differential cross sections.

As a first step, the longitudinal contribution $f_{L}\left(\psi^{\prime}\right)$ obtained in RPWIA and rROP by the Pavia and the Madrid-Sevilla groups at three values of the momentum transfer is displayed in Fig. 5 showing almost coincident results. Similar comments apply to the transverse contribution $f_{T}\left(\psi^{\prime}\right)$. These results, in addition to the cross sections in Fig. 1, confirm the consistency of the numerical codes when calculations are performed under the same conditions.

The scaling function $f_{L}\left(\psi^{\prime}\right)$ evaluated within RPWIA and rROP shows a very mild dependence on the momentum transfer for both positive and negative values of the scaling variable $\psi^{\prime}$, i.e., violation of scaling of first kind is small.

In Figs. 6 and 7 we compare $f_{L}\left(\psi^{\prime}\right)$ evaluated with different models and for several values of the momentum transfer with the averaged QE phenomenological scaling function extracted from the analysis of $\left(e, e^{\prime}\right)$ data [41-43]. As already shown in previous works $[35,44,47]$, the RMF model produces an asymmetric shape with a long tail in the region with $\psi^{\prime}>0$ that follows closely the phenomenological function behavior. The

FIG. 4. Differential cross section of the ${ }^{12} \mathrm{C}\left(e, e^{\prime}\right)$ reaction for different beam energies and electron scattering angles. Line convention as in Fig. 2; experimental data from Refs. [85-87]. 

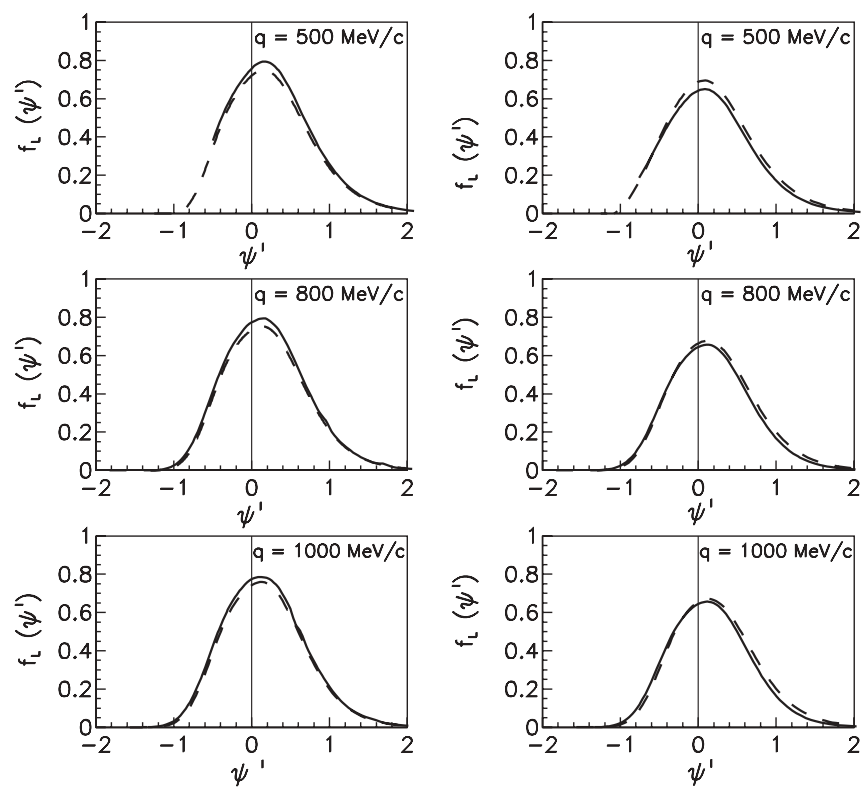

FIG. 5. Longitudinal contribution to the scaling function for three values of the momentum transfer, i.e., $q=500,800$, and $1000 \mathrm{MeV} / c$, obtained by the Pavia (solid lines) and the MadridSevilla (dashed lines) groups with RPWIA (left column) and rROP (right column).

asymmetry in the data has usually been attributed to physical effects beyond the mean field such as two-body currents and short-range correlations [24]. Within a nonrelativistic framework such contributions need to be considered to get asymmetry $[24,88,89]$. By contrast, the RMF approach is capable of explaining the asymmetric behavior of data within
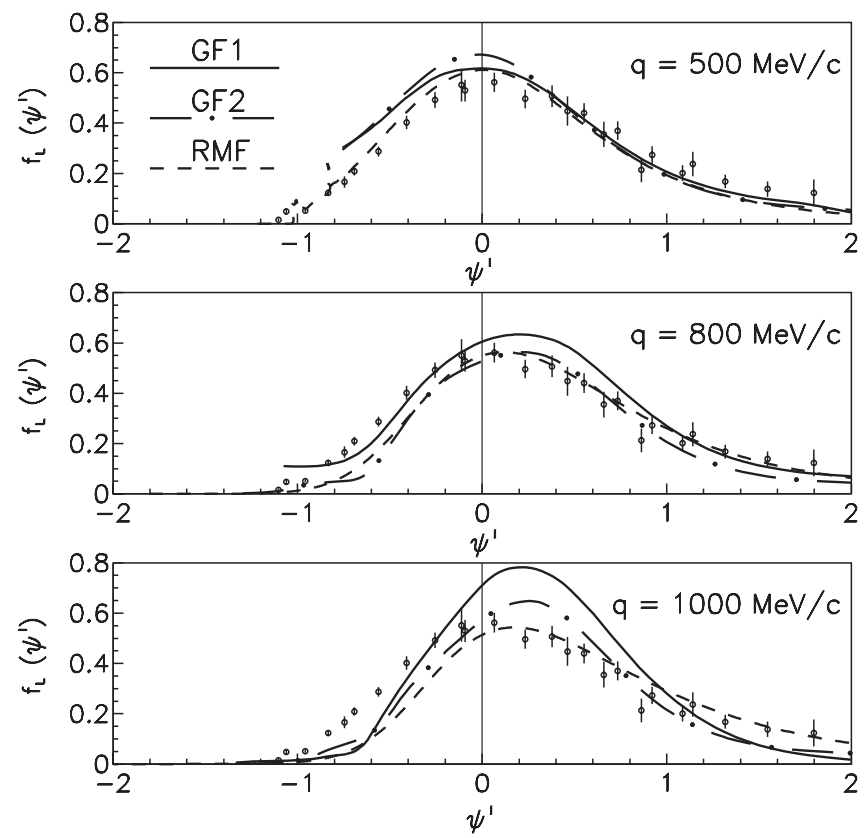

FIG. 6. Longitudinal contribution to the scaling function for $q=$ 500, 800, and $1000 \mathrm{MeV} / c$ with the GF1 (solid), GF2 (long dotdashed), and RMF (dashed) models compared with the averaged experimental scaling function.
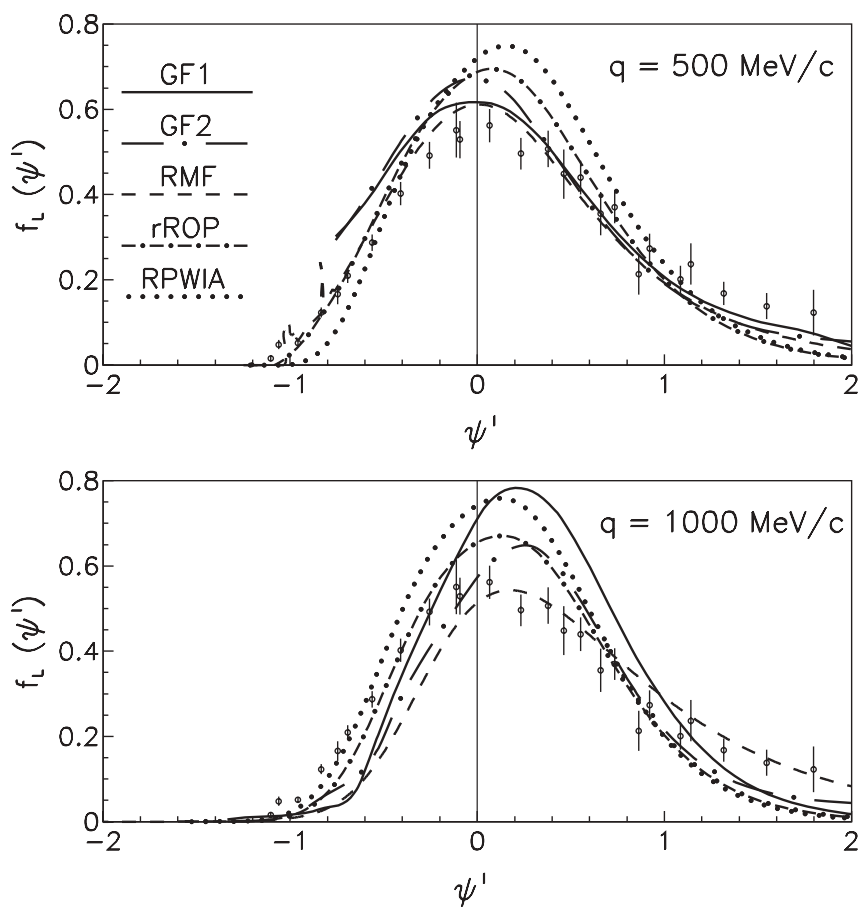

FIG. 7. Longitudinal contributions to the scaling function for $q=500$ and $1000 \mathrm{MeV} / c$ compared with the averaged experimental scaling function. Line convention as in Fig. 3.

the framework of the relativistic impulse approximation taking advantage of its strong relativistic scalar and vector potentials. The results with the GF model are similar to those obtained with $\mathrm{RMF}$ at $q=500 \mathrm{MeV} / c$ and, with moderate differences, at $q=800 \mathrm{MeV} / c$, while visible discrepancies appear at $q=$ $1000 \mathrm{MeV} / c$. However, discussion of results for the scaling functions follows similar trends to the one already applied to the behavior of the cross sections in Fig. 2, i.e., at higher $q$ values the maximum strength occurs for the GF1 model being the RMF one the weakest.

The asymmetric shape with a tail in the region of positive $\psi^{\prime}$ is obtained in both RMF and GF models that involve descriptions of FSI either with a strong energy-independent real potential or with a complex energy-dependent optical potential, respectively. The scaling functions corresponding to RPWIA and rROP, which are also presented in Fig. 7 show no significant asymmetric tail for $\psi^{\prime}>0$. The different dependence on the momentum transfer shown by the potentials involved in the RMF and GF approaches makes the GF scaling function tail less pronounced as the value of $q$ goes up.

The comparison of the different models to the longitudinal scaling function is illuminating. We must recall that the experimental longitudinal response can be considered as a much better representation of the pure nucleonic contribution to the inclusive cross section than the total cross section. It is remarkable that, as seen in Figs. 6 and 7, except for the highest value of $q$ considered $(1000 \mathrm{MeV} / \mathrm{c}), \mathrm{GF} 1$, GF2, and RMF approaches yield very similar predictions for the longitudinal response, in good agreement with the experimental longitudinal response. The asymmetric tail of the data and the strength at the peak are fairly reproduced 

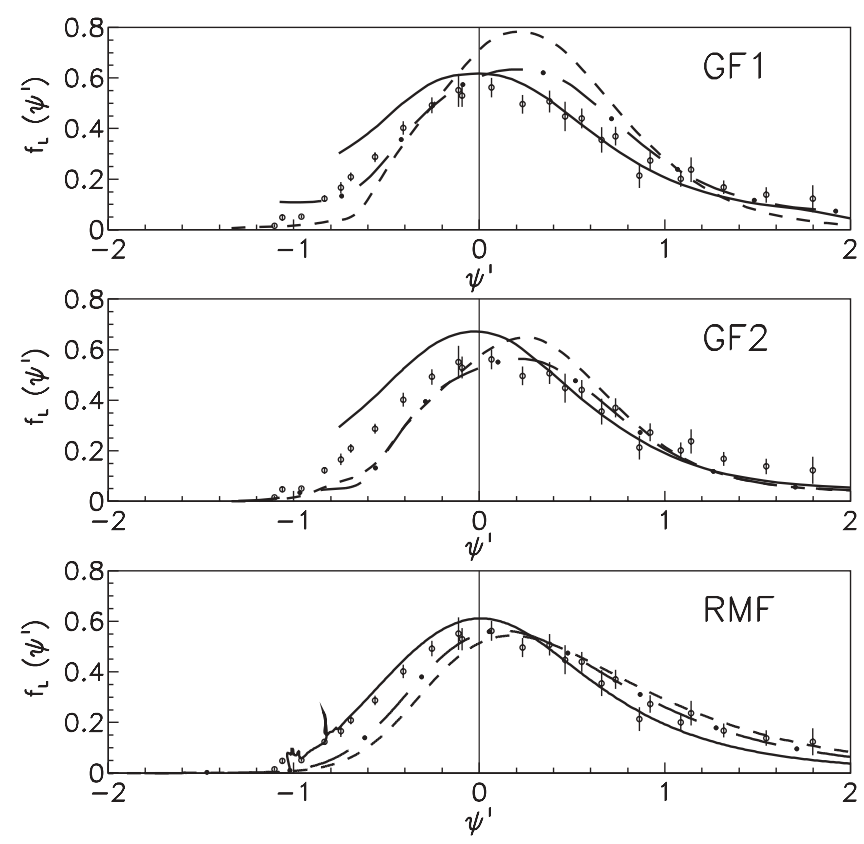

FIG. 8. Analysis of first-kind scaling, $f_{L}\left(\psi^{\prime}\right)$ for $q=500$ (solid) $q=800$ (dot-dashed) and $1000 \mathrm{MeV} / c$ (dashed) with the GF1 (top panel), GF2 (middle panel), and RMF (bottom panel) models using the same results already displayed in Fig. 6.

by the three approaches. However, for $q=1000 \mathrm{MeV} / c$, only the RMF approach seems to be favored from the comparison to the data, while GF1 and GF2 now yield rather different predictions than the RMF approach that seem to be ruled out by data. We must keep in mind that the GF approach uses as input the phenomenological optical potentials. It is clear that, as the momentum transfer increases, the phenomenological optical potential will (implicitily) incorporate a larger amount of contributions from non-nucleonic degrees of freedom, such as for instance the loss of (elastic) flux into the inelastic delta excitation with or without real pion production. Thus the input of the GF formalism is contaminated by not purely nucleonic contributions. Consequently, GF predictions depart from the experimental QE longitudinal response that effectively isolates only nucleonic contributions. This difference, which is larger with increasing momentum transfer, emerges as an excess of strength predicted by the GF model as it translates a loss of flux due to non-nucleonic processes into inclusive purely nucleonic strength. However, the RMF model uses as input the effective mean field that reproduces saturation properties of nuclear matter and of the ground state of the nuclei involved, and thus it is much more suited to estimate purely nucleonic contributions to the inclusive cross section, even at $q=1000 \mathrm{MeV} / c$. Taking these facts into account, the comparison of the models to the data, both for total cross sections and longitudinal responses, yields what one reasonably expects.

An analysis of the scaling of first-kind, i.e., independence of the momentum transfer, is illustrated in Fig. 8. The results are the same already shown in Fig. 6 but are presented in a different way. Each panel corresponds to a specific description of FSI (GF1, GF2, and RMF) and includes results obtained for different values of $q$. The experimental data are compatible with a mild violation of first-kind scaling, particularly in the positive $\psi^{\prime}$ region. In Refs. $[35,44]$ the scaling functions evaluated with the RPWIA and rROP models were shown to depend very mildly on the transferred momentum in the whole, positive and negative, $\psi^{\prime}$ region. In the case of the RMF approach, there is a slight shift in the region $\psi^{\prime}<0$, whereas the model breaks scaling approximately at $30 \%$ level when $\psi^{\prime}>0$. Similar results are obtained with the GF models, where a shift in the region of negative $\psi^{\prime}$ also occurs, and scaling is broken for $\psi^{\prime}>0$. This scaling violation for $\psi^{\prime}>0$ is larger with GF1 due to the enhancement produced in the region of the QE peak by this phenomenological optical potential at higher values of $q$. As a consequence, the comparison between the experimental QE scaling function and the GF1 results is worse than the corresponding comparison for GF2.

\section{SUMMARY AND CONCLUSIONS}

The work developed in this article has emerged as a close collaboration between the Pavia and Madrid-Sevilla groups. For the past several years, both groups have been deeply involved in studies of lepton-scattering reactions with nuclei. Inclusive and exclusive electron-scattering processes have been analyzed, as well as reactions involving the use of neutrinos. The calculations performed by the two groups, which are similar in some aspects, i.e., treatment of relativistic ingredients, bound-nucleon wave functions, single-nucleon current operators, etc., present also clear differences concerning the description of final-state interactions, which constitute an essential ingredient for a successful comparison with data.

The consistency of the numerical calculations developed by our two groups is checked by comparing results in the plane-wave limit (RPWIA) and making use of the real part of the relativistic optical potential (rROP). Almost identical predictions come out within RPWIA and very similar ones with $\mathrm{rROP}$. This reinforces our confidence on the reliability of both calculations. As known, the description of inclusive $\left(e, e^{\prime}\right)$ reactions requires the contribution from the inelastic channels to be retained. Within the framework of the relativistic impulse approximation, a simple recipe to compute the inclusive strength is the use of purely real potentials in the final state. This is the case of the rROP approach (phenomenological relativistic optical potential but without the imaginary part). However, although rROP conserves the flux, its use for inclusive processes is not entirely satisfactory because the (complex) relativistic optical potential has its origin in the description of exclusive processes where only the elastic channel contributes to the observables. Two other models based on the relativistic impulse approximation have been used recently to account for FSI. The former (see Refs. [17,35]) employes distorted waves obtained with the same relativistic mean field considered for the initial bound states. This is denoted as RMF and it fulfills the dispersion relation and the continuity equation. The latter procedure is based on the relativistic Green's function (GF) approach (see Refs. [14,36-39]), which allows one to treat FSI consistently in the inclusive and exclusive reactions. 
Differential cross sections and scaling functions evaluated with both models for different kinematical situations have been compared. Discrepancies are shown to increase with the momentum transfer. This is linked to the energy-dependent optical potentials involved in the relativistic GF method by contrast to the strong energy-independent RMF potentials. Moreover, results presented for two different parametrizations of the ROP, namely EDAD1 vs. EDAD2, prove the importance of the imaginary term, which gets its maximum strength around $500 \mathrm{MeV}$, whereas the real part gets smaller as the energy increases. This explains the different behavior shown by the results of the GF and rROP models, the latter approaching the RPWIA results for higher values of the transferred energy and momentum. Furthermore, discrepancies between the GF and RMF results are also clearly visible as $q$ goes up, due to the contribution from not purely nucleonic inelasticities to the phenomenological optical potential

All models considered respect scaling and superscaling properties. Furthermore, the significant asymmetry in the scaling function produced by the RMF is strongly supported by data [35]. On the contrary, asymmetry is largely absent in RPWIA and rROP predictions. The relativistic GF approach leads to similar results to RMF, i.e., with presence of the asymmetry for intermediate $q$ values. On the contrary, visible discrepancies emerge for larger $q$, being the GF scaling function tail less pronounced but showing more strength in the region where the maximum occurs. Moreover, the GF results for high $q$ present a strong dependence on the specific parametrization considered for the optical potential. These results are directly linked to effects introduced by the imaginary (scalar and vector) term in the optical potential that presents a high sensitivity to the particular ROP parametrization. The relativistic GF approach, even based on the use of a complex optical potential, is shown to preserve flux conservation, hence the imaginary term in the potential leading to a redistribution of the strength among different channels. This explains the difference observed between RMF and GF predictions, the latter with additional strength in the region close to the maximum in the $\mathrm{QE}$ response. This behavior could be connected with effects coming from the contribution of the $\Delta$ that are, somehow, accounted for in a phenomenological way by the GF approach, modifying consequently the responses even in the region where the QE peak gives the main contribution. Note that the higher the transfer momentum the stronger the overlap between the $\mathrm{QE}$ and $\Delta$ peaks. This makes it very difficult to isolate contributions coming from either region.

Although great caution should be exercised in extending the above comments before more conclusive studies are performed, the present analysis can be helpful in disentangling different treatments of FSI and their connections with different physics aspects involved in the process. The similarities of the GF and RMF predictions for the inclusive cross sections, particularly for intermediate values of $q$, in spite of the very different phenomenological ingredients they consider, and the very reasonable agreement with the data for the longitudinal scaled response, that constitutes a good representation of the experimentally measured purely nucleonic response to the inclusive cross sections, are a clear indication of the fact that both models make a very decent job in estimating the inclusive contribution. It will be interesting to investigate the possibility of disentangling in the phenomenological optical potential the contributions due to non-nucleonic inelasticities and extract a "purely nucleonic" optical potential that could then be used in the GF approach and contrasted against the experimental longitudinal scaling function. This work can be considered a first step in this direction.

\section{ACKNOWLEDGMENTS}

We are grateful to M. B. Barbaro for useful discussions and for her valuable advice. This work was partially supported by DGI (Spain) under contract nos. FPA2006-13807-C0201, FPA2007-62216, and FIS2008-04189 and the Spanish Consolider-Ingenio 2010 programme CPAN (CSD200700042). J.A.C. acknowledges support from the INFN-MEC agreement, project "Study of relativistic dynamics in neutrino and electron scattering."
[1] S. Boffi, C. Giusti, F. D. Pacati, and M. Radici, Oxford Studies in Nuclear Physics: Electromagnetic Response of Atomic Nuclei (Clarendon Press, Oxford, 1996), Vol. 20; S. Boffi, C. Giusti, and F. D. Pacati, Phys. Rep. 226, 1 (1993).

[2] O. Benhar, D. Day, and I. Sick, Rev. Mod. Phys. 80, 189 (2008).

[3] J. Jourdan, Phys. Lett. B353, 189 (1995); Nucl. Phys. A603, 117 (1996).

[4] J. J. Kelly, Adv. Nucl. Phys. 23, 75 (1996).

[5] J. D. Walecka, Camb. Monogr. Part. Phys. Nucl. Phys. Cosmol. 16, 1 (2001).

[6] C. R. Chinn, A. Picklesimer, and J. W. Van Orden, Phys. Rev. C 40, 790 (1989).

[7] J. M. Udías, P. Sarriguren, E. Moya de Guerra, E. Garrido, and J. A. Caballero, Phys. Rev. C 48, 2731 (1993); 51, 3246 (1995).

[8] J. M. Udías, P. Sarriguren, E. Moya de Guerra, and J. A. Caballero, Phys. Rev. C 53, R1488 (1996).

[9] J. M. Udías, J. A. Caballero, E. Moya de Guerra, J. R. Vignote, and A. Escuderos, Phys. Rev. C 64, 024614 (2001).
[10] A. Meucci, C. Giusti, and F. D. Pacati, Phys. Rev. C 64, 014604 (2001).

[11] A. Meucci, C. Giusti, and F. D. Pacati, Phys. Rev. C 64, 064615 (2001).

[12] A. Meucci, Phys. Rev. C 65, 044601 (2002).

[13] M. Radici, A. Meucci, and W. H. Dickhoff, Eur. Phys. J. A 17, 65 (2003).

[14] A. Meucci, C. Giusti, and F. D. Pacati, Nucl. Phys. A756, 359 (2006).

[15] Y. Jin, D. S. Onley, and L. E. Wright, Phys. Rev. C 45, 1333 (1992)

[16] K. S. Kim and L. E. Wright, Phys. Rev. C 68, 027601 (2003); 72, 064607 (2005).

[17] C. Maieron, M. C. Martínez, J. A. Caballero, and J. M. Udías, Phys. Rev. C 68, 048501 (2003).

[18] J. W. Van Orden and T. W. Donnelly, Ann. Phys. 131, 451 (1981).

[19] F. Gross and D. O. Riska, Phys. Rev. C 36, 1928 (1987). 
[20] M. B. Barbaro, J. A. Caballero, T. W. Donnelly, and C. Maieron, Phys. Rev. C 69, 035502 (2004).

[21] J. E. Amaro, M. B. Barbaro, J. A. Caballero, T. W. Donnelly, and A. Molinari, Nucl. Phys. A697, 388 (2002).

[22] J. E. Amaro, M. B. Barbaro, J. A. Caballero, T. W. Donnelly, and A. Molinari, Phys. Rep. 368, 317 (2002).

[23] J. E. Amaro, M. B. Barbaro, J. A. Caballero, T. W. Donnelly, and A. Molinari, Nucl. Phys. A723, 181 (2003).

[24] A. De Pace, M. Nardi, W. M. Alberico, T. W. Donnelly, and A. Molinari, Nucl. Phys. A726, 303 (2003); A741, 249 (2004).

[25] G. Benenti, C. Giusti, and F. D. Pacati, Nucl. Phys. A574, 716 (1994).

[26] C. Giusti and F. D. Pacati, Phys. Rev. C 67, 044601 (2003).

[27] A. Meucci, C. Giusti, and F. D. Pacati, Phys. Rev. C 66, 034610 (2002).

[28] J. P. McDermott, E. Rost, J. R. Shepard, and C. Y. Cheung, Phys. Rev. Lett. 61, 814 (1988).

[29] J. Ryckebusch, K. Heyde, L. Machenil, D. Ryckbosch, M. Vanderhaeghen, and M. Waroquier, Phys. Rev. C 46, R829 (1992).

[30] T. B. Bright and S. R. Cotanch, Phys. Rev. Lett. 71, 2563 (1993).

[31] T. B. Bright, B. Hölstad, R. Johansson, E. Traneus, and S. R. Cotanch, Nucl. Phys. A603, 1 (1996).

[32] M. Anguiano, G. Co', A. M. Lallena, and S. R. Mokhtar, Ann. Phys. 296, 235 (2002).

[33] J. M. Udías, J. A. Caballero, E. Moya de Guerra, J. E. Amaro, and T. W. Donnelly, Phys. Rev. Lett. 83, 5451 (1999).

[34] M. C. Martínez, J. R. Vignote, J. A. Caballero, T. W. Donnelly, E. Moya de Guerra, and J. M. Udías, Phys. Rev. C 69, 034604 (2004).

[35] J. A. Caballero, Phys. Rev. C 74, 015502 (2006).

[36] A. Meucci, F. Capuzzi, C. Giusti, and F. D. Pacati, Phys. Rev. C 67, 054601 (2003).

[37] A. Meucci, C. Giusti, and F. D. Pacati, Nucl. Phys. A739, 277 (2004).

[38] F. Capuzzi, C. Giusti, and F. D. Pacati, Nucl. Phys. A524, 681 (1991).

[39] F. Capuzzi, C. Giusti, F. D. Pacati, and D. N. Kadrev, Ann. Phys. 317, 492 (2005)

[40] D. B. Day, J. S. McCarthy, T. W. Donnelly, and I. Sick, Annu. Rev. Nucl. Part. Sci. 40, 357 (1990).

[41] C. Maieron, T. W. Donnelly, and I. Sick, Phys. Rev. C 65, 025502 (2002).

[42] T. W. Donnelly and I. Sick, Phys. Rev. Lett. 82, 3212 (1999).

[43] T. W. Donnelly and I. Sick, Phys. Rev. C 60, 065502 (1999).

[44] J. A. Caballero, J. E. Amaro, M. B. Barbaro, T. W. Donnelly, C. Maieron, and J. M. Udías, Phys. Rev. Lett. 95, 252502 (2005).

[45] J. E. Amaro, M. B. Barbaro, J. A. Caballero, T. W. Donnelly, A. Molinari, and I. Sick, Phys. Rev. C 71, 015501 (2005).

[46] M. V. Ivanov, M. B. Barbaro, J. A. Caballero, A. N. Antonov, E. Moya de Guerra, and M. K. Gaidarov, Phys. Rev. C 77, 034612 (2008).

[47] J. A. Caballero, J. E. Amaro, M. B. Barbaro, T. W. Donnelly, and J. M. Udías, Phys. Lett. B653, 366 (2007).

[48] J. E. Amaro, M. B. Barbaro, J. A. Caballero, T. W. Donnelly, and C. Maieron, Phys. Rev. C 71, 065501 (2005).

[49] A. N. Antonov, M. V. Ivanov, M. K. Gaidarov, E. Moya de Guerra, J. A. Caballero, M. B. Barbaro, J. M. Udías, and P. Sarriguren, Phys. Rev. C 74, 054603 (2006).

[50] M. Martini, G. Co', M. Anguiano, and A. M. Lallena, Phys. Rev. C 75, 034604 (2007).
[51] J. E. Amaro, M. B. Barbaro, J. A. Caballero, and T. W. Donnelly, Phys. Rev. C 73, 035503 (2006).

[52] M. C. Martínez, J. A. Caballero, T. W. Donnelly, and J. M. Udías, Phys. Rev. Lett. 100, 052502 (2008); Phys. Rev. C 77, 064604 (2008)

[53] A. N. Antonov, M. V. Ivanov, M. B. Barbaro, J. A. Caballero, E. Moya de Guerra, and M. K. Gaidarov, Phys. Rev. C 75, 064617 (2007).

[54] J. E. Amaro, M. B. Barbaro, J. A. Caballero, T. W. Donnelly, and J. M. Udías, Phys. Rev. C 75, 034613 (2007).

[55] Y. Horikawa, F. Lenz, and N. C. Mukhopadhyay, Phys. Rev. C 22, 1680 (1980).

[56] M. C. Martínez, P. Lava, N. Jachowicz, J. Ryckebusch, K. Vantournhout, and J. M. Udías, Phys. Rev. C 73, 024607 (2006).

[57] P. Lava, M. C. Martínez, J. Ryckebusch, J. A. Caballero, and J. M. Udías, Phys. Lett. B595, 177 (2004).

[58] A. Meucci, C. Giusti, and F. D. Pacati, Nucl. Phys. A773, 250 (2006).

[59] A. Meucci, C. Giusti, and F. D. Pacati, Phys. Rev. C 77, 034606 (2008).

[60] A. V. Butkevich and S. A. Kulagin, Phys. Rev. C 76, 045502 (2007).

[61] A. V. Butkevich, arXiv:0904.1472 [nucl-th].

[62] O. Benhar and D. Meloni, Nucl. Phys. A789, 379 (2007).

[63] T. Leitner, L. Alvarez-Ruso, and U. Mosel, Phys. Rev. C 73, 065502 (2006).

[64] O. Buss, T. Leitner, U. Mosel, and L. Alvarez-Ruso, Phys. Rev. C 76, 035502 (2007).

[65] T. Leitner, O. Buss, L. Alvarez-Ruso, and U. Mosel, Phys. Rev. C 79, 034601 (2009).

[66] International Workshop on Neutrino-Nucleus Interactions in the Few-GeV Region (NuInt), conference series 2001-2009, see, e.g., http://nuint09.ifae.es/Welcome.html.

[67] K2K, http://neutrino.kek.jp/.

[68] MiniBooNE, http://www-boone.fnal.gov/.

[69] SciBooNE, http://www-sciboone.fnal.gov/.

[70] MicroBooNE, http://www-microboone.fnal.gov/.

[71] T2K, http://jnusrv01.kek.jp/public/t2k/.

[72] MINOS, http://www-numi.fnal.gov/.

[73] MINERvA, http://minerva.fnal.gov/.

[74] NOvA, http://www-nova.fnal.gov/.

[75] T. de Forest, Jr., Nucl. Phys. A392, 232 (1983).

[76] S. Galster et al., Nucl. Phys. B32, 221 (1971).

[77] C. J. Horowitz and B. D. Serot, Nucl. Phys. A368, 503 (1981); Phys. Lett. B86, 146 (1979).

[78] B. D. Serot and J. D. Walecka, Adv. Nucl. Phys. 16, 1 (1986).

[79] W. Pöschl, D. Vretenar, and P. Ring, Comput. Phys. Commun. 103, 217 (1997).

[80] G. A. Lalazissis, J. König, and P. Ring, Phys. Rev. C 55, 540 (1997).

[81] M. M. Sharma, M. A. Nagarajan, and P. Ring, Phys. Lett. B312, 377 (1993).

[82] B. C. Clark, in Proceedings of the Workshop on Relativistic Dynamics and Quark-Nuclear Physics, edited by M. B. Johnson and A. Picklesimer (John Wiley \& Sons, New York, 1986), p. 302; E. D. Cooper, S. Hama, B. C. Clark, and R. L. Mercer, Phys. Rev. C 47, 297 (1993).

[83] M. B. Barbaro, R. Cenni, A. De Pace, T. W. Donnelly, and A. Molinari, Nucl. Phys. A643, 137 (1998). 
[84] L. Alvarez-Ruso, M. B. Barbaro, T. W. Donnelly, and A. Molinari, Nucl. Phys. A724, 157 (2003).

[85] P. Barreau et al., Nucl. Phys. A402, 515 (1983).

[86] D. B. Day et al., Phys. Rev. C 48, 1849 (1993).

[87] R. Sealock et al., Phys. Rev. Lett. 62, 1350 (1989).
[88] C. Bleve, G. Co', I. De Mitri, P. Bernardini, G. Mancarella, D. Martello, and A. Surdo, Astropart. Phys. 16, 145 (2001); G. Co', C. Bleve, I. De Mitri, and D. Martello, Nucl. Phys. Proc. Suppl. 112, 210 (2002).

[89] J. Nieves, J. E. Amaro, and M. Valverde, Phys. Rev. C 70, 055503 (2004). 